\title{
The Wavelength-Locking of High-Power 808 nm Semiconductor Laser
}

\author{
Hai-Xia Guo, Zai-Jin Li, Hui Li, Feng Gao, Hai-Bin Tang, Bao-Hua Shi, and Yi Qu \\ National Key Lab of High Power Semiconductor Lasers, Changchun University of Science and Technology, Changchun, \\ Jilin 130022, China \\ Correspondence should be addressed to Yi Qu; quyi@cust.edu.cn
}

Received 27 July 2014; Accepted 15 September 2014

Academic Editor: Stephen D. Prior

Copyright (C) 2015 Hai-Xia Guo et al. This is an open access article distributed under the Creative Commons Attribution License, which permits unrestricted use, distribution, and reproduction in any medium, provided the original work is properly cited.

\begin{abstract}
A distributed feedback (DFB) laser of $808 \mathrm{~nm}$ is produced in this paper whose optical power is $2 \mathrm{~W}$, cavity length is $3 \mathrm{~mm}$, and injecting width is $200 \mu \mathrm{m}$. A second-order grating formed into an InGaP/GaAs/InGaP multilayer structure provides the optical distributed feedback. The holographic lithography method is adopted to make Bragg gratings in p-waveguide layer $(\Lambda=240 \mathrm{~nm})$ of the GaAs epitaxial wafers. The best experimental conditions are determined by analyzing the surface morphology and threedimensional holographic grating. In addition, the output power data and wavelength of the distributed feedback laser emitting at different temperatures are presented. And the wavelength varies with temperature at a rate of $0.062 \mathrm{~nm} / \mathrm{K}$. Finally, the conclusion is drawn that this kind of DFB laser has a better temperature stabilized wavelength and narrower line width.
\end{abstract}

\section{Introduction}

Semiconductor diode laser emitting at $808 \mathrm{~nm}$ wavelength is of particular interest for pumping $\mathrm{Nd}$ - and $\mathrm{Yb}$-doped solid state gain media, respectively [1]. The semiconductor diode laser pumps for solid-state lasers have to be automatically temperature controlled to ensure that no appreciable detuning occurs between the center of the pump wavelength and the absorption peak. The stable output characteristics can be acquired by using some wavelength-locked devices or an etalon in the feedback loop of light. By acting as a wavelength-locked device in a vertical-cavity surfaceemitting laser (VCSELS) the volume holographic gratings (VHGS) which are produced outside the laser cavity can keep a narrow spectral response and a small spatial acceptance angle analysis in [2]. But the shortcomings of large size and expensive preparation of VCSELS have confined the scope of applications. Another possibility is the use of an internal Bragg grating produced into the laser cavity. This method can also satisfy the requirements of temperature stabilized wavelength and narrow line width. These kinds of devices are called distributed feedback (DFB) lasers or distributed Bragg reflector (DBR) lasers.
Figure 1 shows the differences between DBR lasers and DFB lasers. As is depicted in Figure 1(a), DBR laser consists of a passive DBR section and an active gain section. The DBR section is produced at the rear side of the cavity. However Fricke et al. [3] tell us that owing to the longitudinal mode requirement DBR laser has a nonlinearity light-current characteristic. A DFB laser is shown in Figure 1(b); a Bragg grating is placed in the active gain section or in p-waveguide layer of the epitaxial wafers. The second-order grating acting as a resonant cavity provides both optical distributed feedback and light amplification. In this structure, the wavelength varies with injection current, and the variation is governed by the rate of wavelength that varies with the temperature. DFB lasers with this structure can acquire a linear light-current characteristic in a longitudinal mode.

In the 1970s, Nakemurra made a DFB laser with periodic grating for the first time. In the last century, the development of DFB laser gradually became mature. It is widely used in wavelength division multiplexing (WDM) and dense wavelength division multiplexing (DWDM) communication system. Laser pumping is a large market segment for highpower diode lasers in recent years, and $808 \mathrm{~nm}$ is the most widespread pump wavelength. These two reasons lead to 


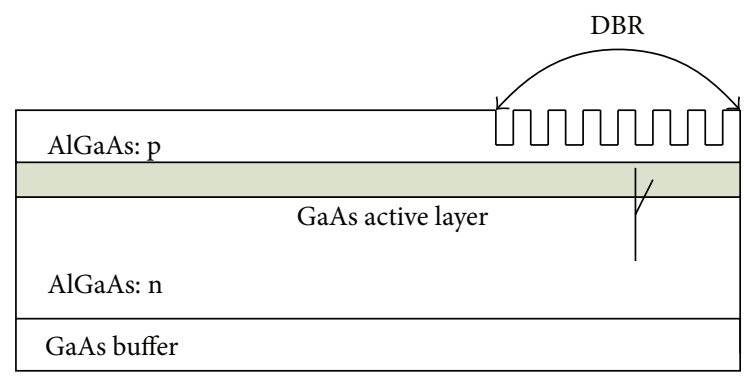

(a)

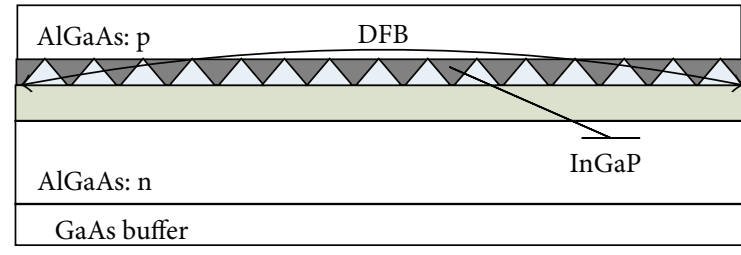

(b)

FIGURE 1: The structures of DBR and DFB lasers' wafers.

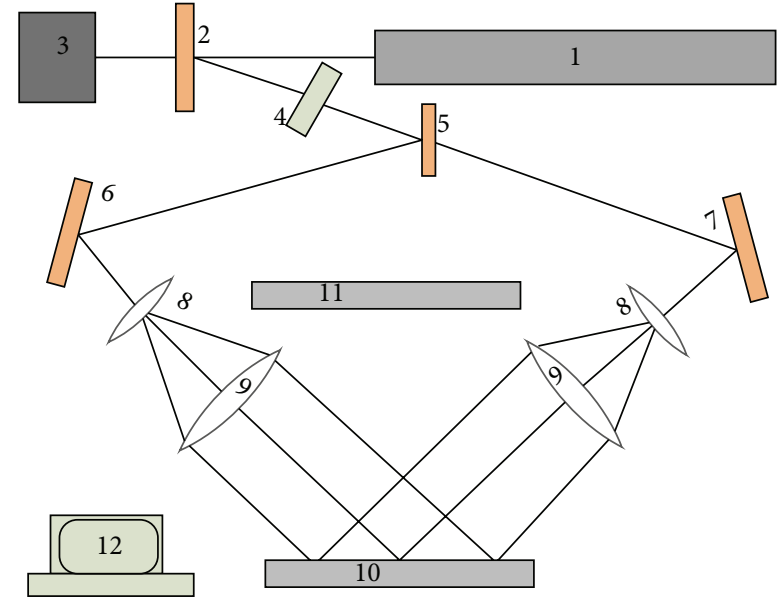

FIGURE 2: Holographic optical path of the exposure. (1) $325 \mathrm{~nm}$ laser; (2) sampling mirror; (3) power detector; (4) exposure switch; (5) half mirror; (6) piezoelectric ceramic reflector; (7) total reflection mirror; (8) UV beam expander; (9) collimating lens; (10) workpiece support; (11) CCD; (12) control computer.

a new wave of research about $808 \mathrm{~nm}$ DFB semiconductor laser. In the late 2010s and early 2005s, in QPC and Alfalight Inc., $808 \mathrm{~nm}$ DFB semiconductor lasers were developed. They reported that the temperature dependence of the spectrum was measured between $0.07 \mathrm{~nm} / \mathrm{K}$ and $0.08 \mathrm{~nm} / \mathrm{K}$. In this paper, a DFB laser operating at $808 \mathrm{~nm}$ is presented with the rate of lasting output wavelength along with temperature variation of $0.062 \mathrm{~nm} / \mathrm{K}$.

\section{Materials and Methods}

The whole preparation of the DFB laser wafer can be divided into three steps: the first epitaxial growth, grating preparation, and the second epitaxial growth. The first growth consists of $0.5 \mu \mathrm{m} \mathrm{N}$-GaAs (Si-doped) buffer, $0.05 \mu \mathrm{m}$ $\mathrm{N}-\mathrm{Al}_{x} \mathrm{Ga}_{1-x} \mathrm{As}(x=0.05-0.55$, Si-doped) gradient transition layer, $1.2 \mu \mathrm{m} \mathrm{N}-\mathrm{Al}_{0.55} \mathrm{Ga}_{0.45}$ As (Si-doped) confinement layer, $0.15 \mu \mathrm{m} \mathrm{Al}_{x} \mathrm{Ga}_{1-x} \mathrm{As}$ ( $x=0.55-0.25$ ) gradient waveguide layer, $5 \mathrm{~nm} \mathrm{Al}_{0.25} \mathrm{Ga}_{0.75}$ As quantum well barrier layer, $4 \mathrm{~nm} \mathrm{Al} 0_{0.07} \mathrm{Ga}_{0.93}$ As quantum well layer, $5 \mathrm{~nm} \mathrm{Al}_{0.25} \mathrm{Ga}_{0.75} \mathrm{As}$

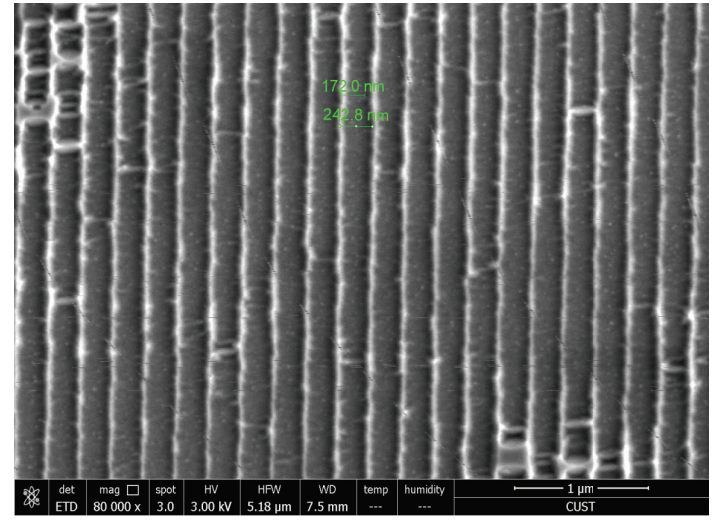

FIGURE 3: SEM image of the grating.

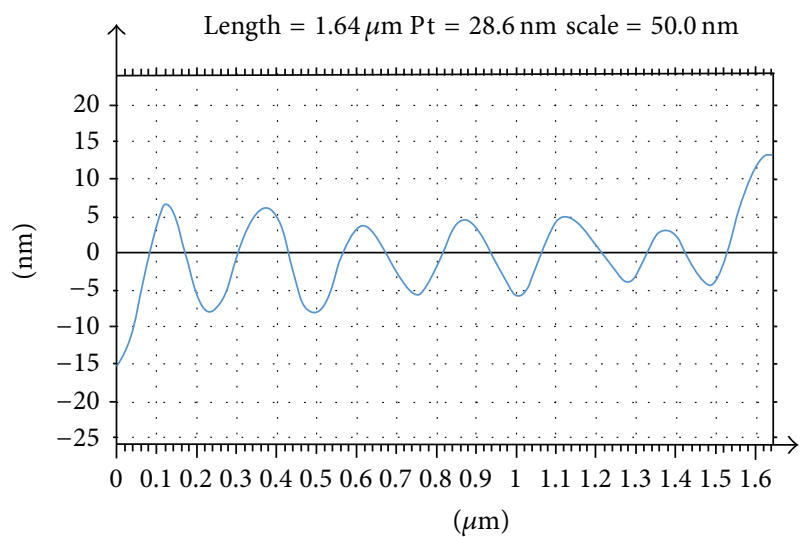

FIGURE 4: The graph of the depth of the grating.

quantum well barrier layer, and $0.15 \mu \mathrm{m} \mathrm{Al}_{x} \mathrm{Ga}_{1-x} \mathrm{As}$ ( $x=$ $0.25-0.55)$ gradient waveguide layer and an InGaP grating layer was prepared by metal-organic chemical vapor deposition (MOCVD). The holographic lithography and wet-chemical etching technology were utilized to produce holographic grating in the InGaP grating layer. The secondorder grating was produced using an exposure system with a $325 \mathrm{~nm}$ He-Cd semiconductor laser. Figure 2 shows the holographic optical path of the exposure. The light path includes 


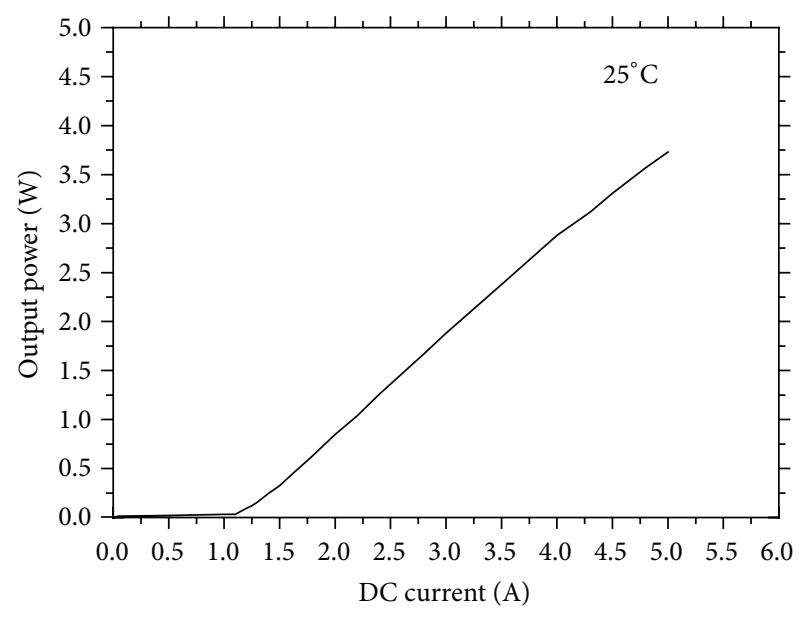

(a)

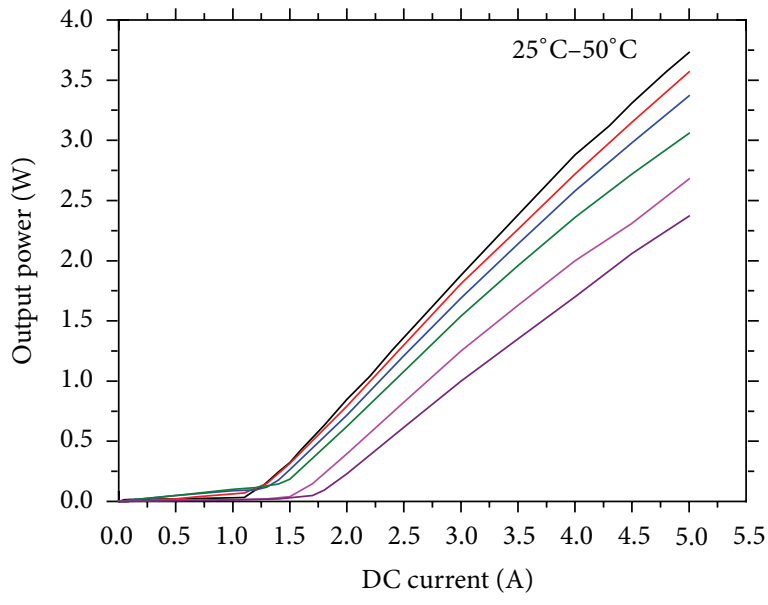

(b)

Figure 5: (a) Light-current characteristics of the DFB laser at the heat-sink temperature of $25^{\circ} \mathrm{C}$. (b) Light-current characteristics of the DFB laser at different temperatures (increment $5 \mathrm{~K}$ ).

a half mirror, a total reflection mirror, and a control system. The thickness of the photoresist is $120 \mathrm{~nm}$. The exposure power is $50 \mathrm{~mW}$, the exposure time is $160 \mathrm{~s}$, and the development time is $6 \mathrm{~s}$. Then the etchant is used to etch lithographic sheet; the etching time is $40 \mathrm{~s}$. After surface cleaning, in the second epitaxial growth the remainder of $0.15 \mu \mathrm{m} \mathrm{Al} \mathrm{Ga}_{1-x}$ As $(x=0.25-0.55)$ gradient waveguide layer, $0.1 \mu \mathrm{m}$ P-Al ${ }_{0.55} \mathrm{Ga}_{0.5}$ As (Zn-doped) buffer layer, $1.2 \mu \mathrm{m}$ P-Al ${ }_{0.55} \mathrm{Ga}_{0.45}$ As (Zn-doped) confinement layer, $0.05 \mu \mathrm{m}$ $\mathrm{N}-\mathrm{Al}_{x} \mathrm{Ga}_{1-x}$ As ( $\left.x=0.55-0.65\right)$ (Zn-doped) gradient transition layer, and a $0.3 \mu \mathrm{m} \mathrm{p}$-GaAs (Zn-doped) contact layer were grown.

\section{Results and Discussion}

We produced the epitaxial wafer which was prepared by using the above experimental conditions and technique into a DFB laser. The DFB laser's optical power is $2 \mathrm{~W}$, cavity length is $3 \mathrm{~mm}$, and injecting width is $200 \mu \mathrm{m}$. The optical power at different heat-sink temperatures is measured utilizing the power detector.

Figures 3 and 4 show the scanning electron microscope (SEM) image and the AFM image of the grating. According to the relation

$$
\sigma=\frac{b}{\Lambda}
$$

where $\sigma$ is the duty cycle, $b$ is the slit spacing of grating, and $\Lambda$ is the period of grating. According to Figure 3, we can find that $b=70.8 \mathrm{~nm}, \Lambda=242.8 \mathrm{~nm}$, and $\sigma \approx 0.29$; the grating has uniformly distributed stripes, good surface morphology, and excellent coupling characteristics. The depth of the grating is about $20 \mathrm{~nm}$. This is a second-order grating; it can eliminate degenerate modes which the first-order grating generates, output single mode, and improve the characteristics of the light beam. The above data were determined resulting in a coupling coefficient $\kappa L$ of value of only 1 .
Figure 5 shows light-current characteristics of the $808 \mathrm{~nm}$ DFB laser at different temperatures. The threshold current is $0.52 \mathrm{~A}$ and the slope efficiency is $0.67 \mathrm{~W} / \mathrm{A}$ near threshold at the heat-sink temperature $T$ of $25^{\circ} \mathrm{C}$. This efficiency is the result of the coupling coefficient $\kappa$ of the Bragg grating. Characteristic temperature can be used to describe the effect of operating characteristics of semiconductor laser on temperature, according to the relation

$$
T=\frac{T_{1}-T_{2}}{\ln \left(I_{\mathrm{th}}\left(T_{1}\right) / I_{\mathrm{th}}\left(T_{2}\right)\right)},
$$

where $T$ is the characteristic temperature, $I_{\mathrm{th}}\left(T_{1}\right)$ is the threshold current at the temperature of $T_{1}$, and $I_{\mathrm{th}}\left(T_{2}\right)$ is the threshold current at the temperature of $T_{2}$. As discussed by Schultz et al. [4] the larger $T$ is, the better temperature characteristics of the device will be. In this paper the characteristic temperature $(T)$ is $77 \mathrm{~K}$. This DFB laser's characteristic temperature is significantly lower than conventional lasers.

Figure 6 shows the optical spectra of the DFB laser in the temperature range of $20^{\circ} \mathrm{C} \sim 55^{\circ} \mathrm{C}$. A wavelength spacing of approximately $0.98 \mathrm{~nm}$ is observed between the main peaks. The output wavelengths were found to be in the range from $807 \mathrm{~nm}$ to $809 \mathrm{~nm}$. Figure 7 shows the wavelength shift of the DFB laser at different heat-sink temperatures. We found that the rate of lasting output wavelength along with temperature variation is $0.062 \mathrm{~nm} / \mathrm{K}$. The rate is lower than $0.067 \mathrm{~nm} / \mathrm{K}$ in [5]. The output wavelength has been locked; the Bragg wavelength and the output spectrum have been narrowed for a wide range of heat-sink temperatures. Without the Bragg gratings, the output wavelength of the laser will be very dependent. So the Bragg gratings are effective to overcome the wavelength shift.

\section{Conclusions}

The wavelength of the $808 \mathrm{~nm}$ laser was locked by producing the Bragg grating into the $\mathrm{InGaP} / \mathrm{GaAs} / \mathrm{InGaP}$ multilayer 


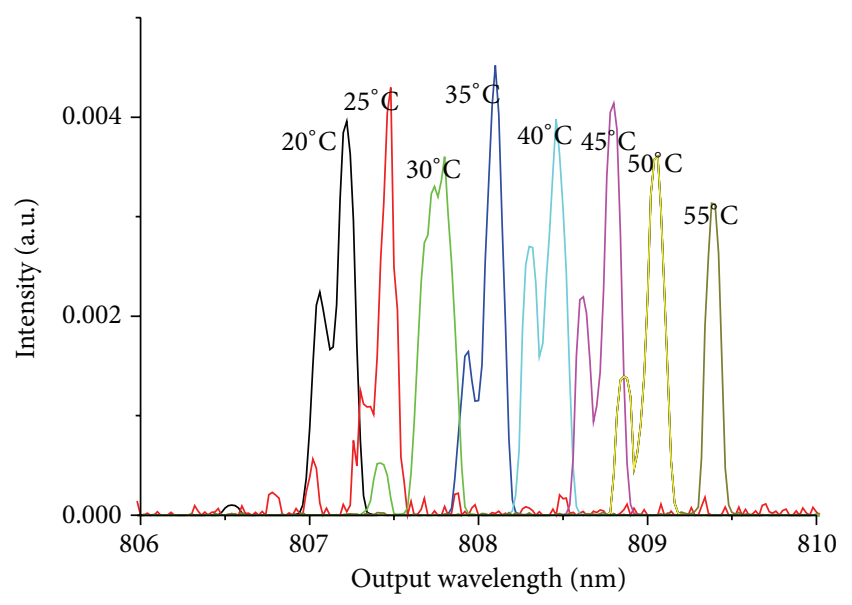

FIgURE 6: Optical spectra of DFB laser at different temperatures (increment $5 \mathrm{~K}$ ).

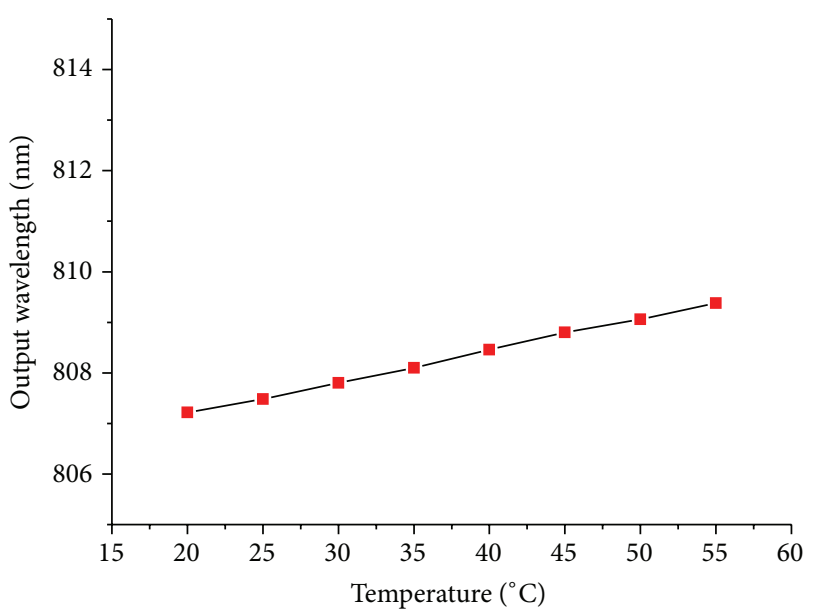

Figure 7: The wavelength shift of the DFB laser.

structure. Then a laser with single longitudinal mode and narrow injecting width was acquired. For lasers with different wavelengths, the grating period can be changed to achieve wavelength-locking. So this method can be widely used in almost all sorts of lasers and the holographic lithography method can be adopted to make Bragg gratings. There is room for optimization of the uniformity of grating with regard to diffraction efficiency, by considering the trade-offs between locking range, output spectrum, and power efficiency.

\section{Conflict of Interests}

The authors declare that there is no conflict of interests regarding the publication of this paper.

\section{Acknowledgments}

The authors are grateful to the National Key Lab of High Power Semiconductor Lasers and Changchun University of
Science and Technology for technical assistance. This paper was supported by NSAF, Grant no. U1330136.

\section{References}

[1] S. Li, Y. Shi, J. Li, and R. Gu, "Experimental demonstration of the corrugation pitch modulated DFB semiconductor laser based on the reconstruction-equivalent-chirp technology," in Proceedings of the Asia Communications and Photonics Conference and Exhibition (ACP '10), pp. 112-113, Shanghai, China, 2010.

[2] G. J. Steckman, W. Liu, R. Platz, D. Schroeder, C. Moser, and F. Havermeyer, "Volume holographic grating wavelength stabilized laser diodes," IEEE Journal on Selected Topics in Quantum Electronics, vol. 13, no. 3, pp. 672-678, 2007.

[3] J. Fricke, F. Bugge, A. Ginolas et al., "High-power 980-nm broad-area lasers spectrally stabilized by surface bragg gratings," IEEE Photonics Technology Letters, vol. 22, no. 5, pp. 284286, 2010.

[4] C. M. Schultz, P. Crump, H. Wenzel et al., "11W broad area $976 \mathrm{~nm}$ DFB lasers with 58\% power conversion efficiency," Electronics Letters, vol. 46, no. 8, pp. 580-581, 2010.

[5] M. Kanskar, H. An, J. Cai, C. Galstad, E. Stiers, and Y. He, SSDLTR, Los Angeles, Calif, USA, 2007. 


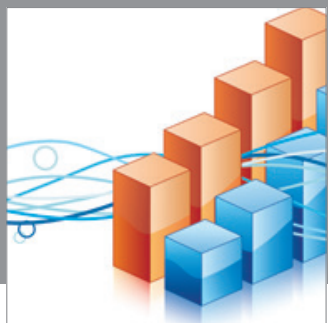

Advances in

Operations Research

mansans

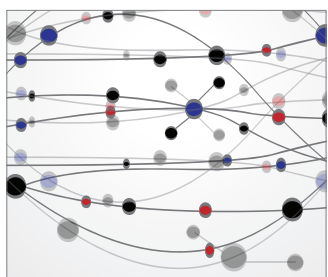

The Scientific World Journal
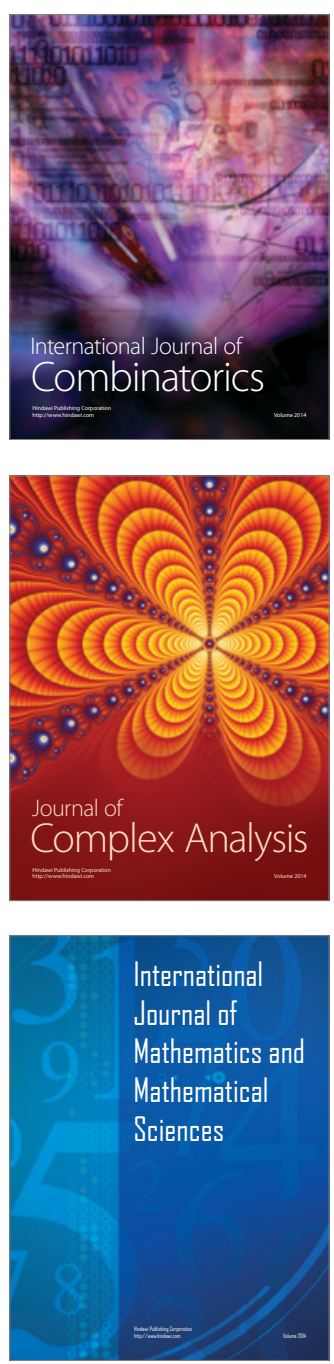
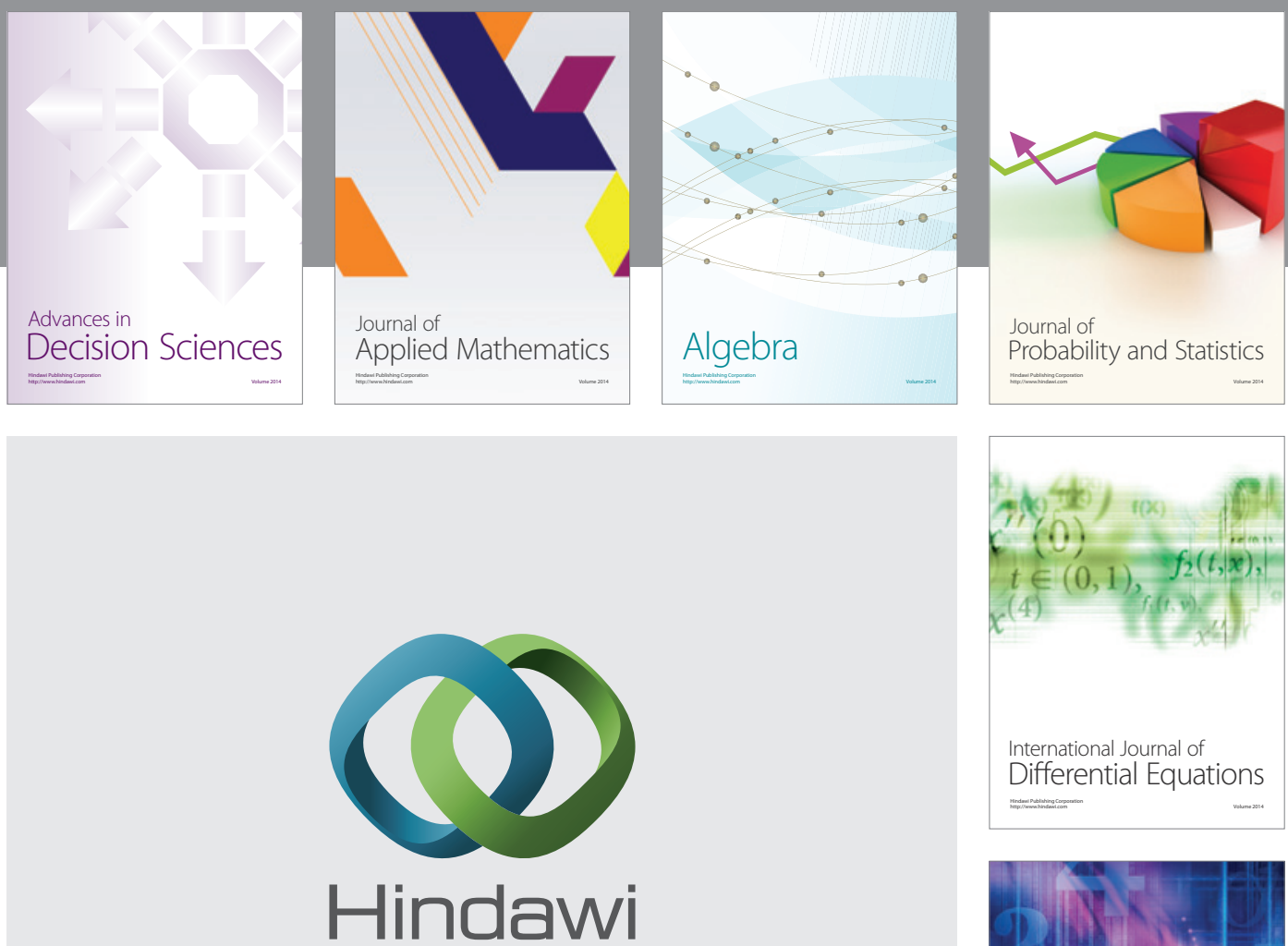

Submit your manuscripts at http://www.hindawi.com
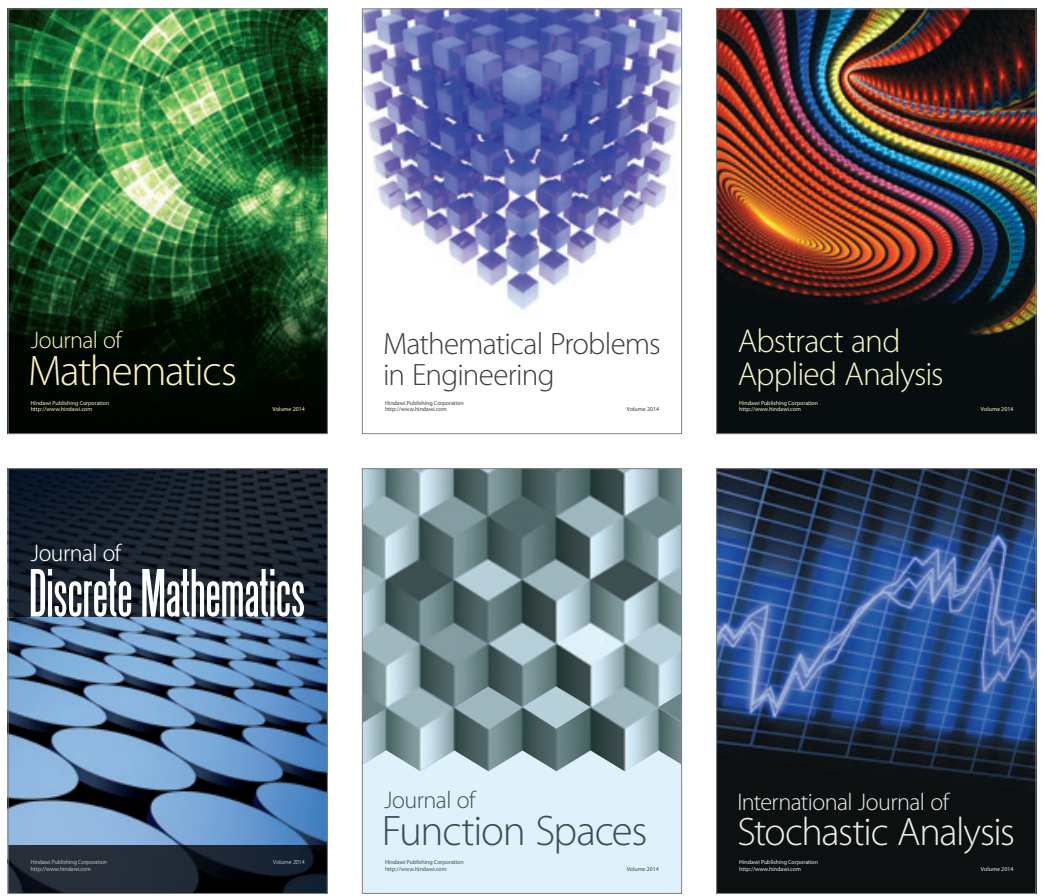

Journal of

Function Spaces

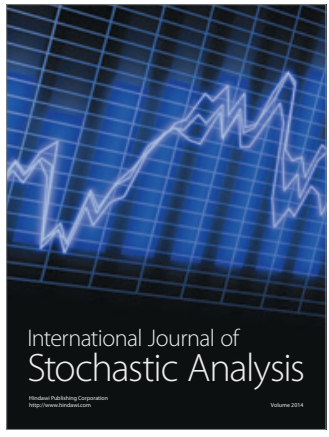

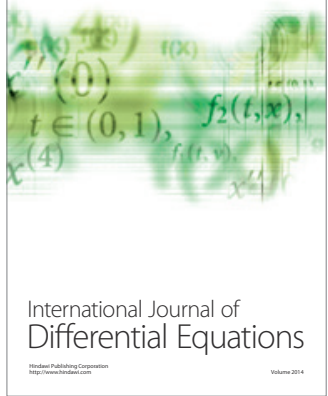
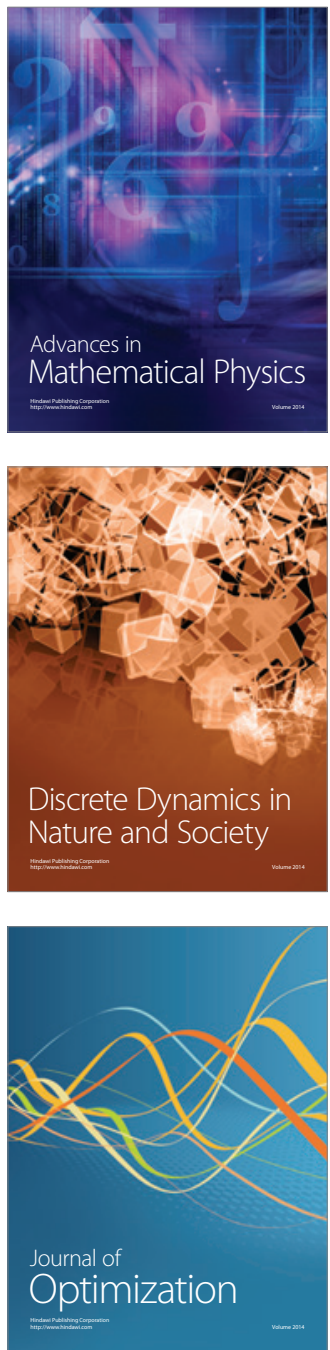